\title{
In Situ 4D-STEM
}

\section{Andrew Minor}

UC Berkeley and LBNL, Berkeley, California, United States

In situ transmission electron microscopy (TEM) experiments are typically recorded either in real space or diffraction space [1]. However, it would be ideal to have both real and diffraction space for when transient events occur that cannot be repeated exactly (ie- defect generation or irreversible phase transformations). Real space imaging provides context for these transient events by spatially-resolving microstructural features to one another while diffraction space provides better structural clarity about phase identification and lattice parameters.

Four-dimensional scanning transmission electron microscopy (4D-STEM) [2], can come close to providing both simultaneous real-space imaging and diffraction analysis during in situ testing. For example, with the advent of fast direct electron detectors it is possible to perform strain mapping via diffraction pattern analysis during in-situ deformation in a TEM with the precision of a few nanometers without stopping the experiment [3,4]. Images of the same overall experiment can then be formed using virtual apertures applied to the accumulated diffraction patterns [5]. The resolution and quality of the reconstructed images in these experiments is typically limited by the size of the probe. For example, in order to form diffraction spot patterns for strain analysis a probe size of $\sim 1 \mathrm{~nm}$ is typical and therefore the virtual bright field or virtual dark field images are not at the same resolution as a typical STEM image or diffraction contrast TEM image. However, the reconstructed virtual image quality is still usually good enough to identify line defects in materials (and even analyze defect character by performing $g$ dot $b$ analysis from a single data cube of diffraction patterns). The frame rate of the virtual images from a 4DTEM experiment is necessarily slower than the maximum speed of the detector, since the frame rate of the images is a function of the camera frame rate, field of view and step size of the probe.

This talk will highlight recent in situ 4DSTEM experiments that explore transient events where both information from diffraction space and real space are used. The diffraction patterns are used to identify different phases, orientations and relative strain, while the images formed by using virtual apertures provide microstructural context for the analysis. Example experiments include defect generation and interactions in metals, [6], local strain evolution in bulk metallic glasses [6,7], and structural transformations in functional oxides under deformation $[8,9,10]$.

\section{References}

[1] F. Ross and A. M. Minor, "In situ Transmission Electron Microscopy", in Springer Handbook of Microscopy, edited by Peter Hawkes and John Spence, Springer Nature Switzerland AG, 2019

[2] C Ophus, Microscopy and Microanalysis 25 (2019) p. 563.

[3] V. B. Ozdol, et al, Applied Physics Letters 106 (2015), p. 253107.

[4] C. Gammer, et al, Applied Physics Letters 109 (2016), p. 081906

[5] C. Gammer, et al., Ultramicroscopy, 155, p. 1-10 (2015)

[6] T. C. Pekin et al, Scripta Materialia 146 (2018), p. 87-90.

[7] C. Gammer, et al. Applied Physics Letters, 112171905 (2018)

[8] Yu Deng, et al., Acta Materialia, 181, 501-509 (2019)

[9] Thomas C. Pekin, et al., Nature Communications, 10 (1) 2445 (2019)

[10] This work was supported by the Director, Office of Science, Office of Basic Energy Sciences, Materials Sciences andEngineering Division, of the U.S. Department of Energy under Contract No. DE- 
AC02-05-CH11231 within the Mechanical Behavior of Materials (KC 13) program at the Lawrence Berkeley National Laboratory, the U.S. Office of Naval Research and STROBE, a Science and Technology Center sponsored by the National Science Foundation. Work at the Molecular Foundry is supported by the Office of Science, Office of Basic Energy Sciences, of the U.S. Department of Energy under Contract No. DE-AC02-05CH11231. 